\title{
Morphokinetic parameters comparison between euploid and aneuploid blastocysts
}

\author{
Phuong Thi Bich Le ${ }^{1,2, *(\infty)}$, Anh Hoang Le ${ }^{1,2}$, Loc Minh Tai Nguyen ${ }^{1,2}$, Vinh Quang Dang ${ }^{1,2}$
}

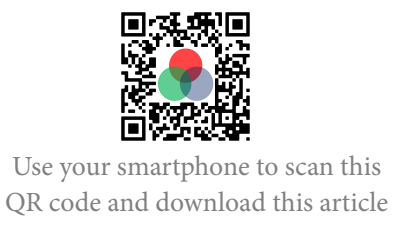

${ }^{1} I V M F D$ PN, My Duc Phu Nhuan Hospital, Ho Chi Minh City, Viet Nam

${ }^{2}$ HOPE Research Center, Ho Chi Minh City, Viet Nam

\section{Correspondence}

Phuong Thi Bich Le, IVMFD PN, My Duc Phu Nhuan Hospital, Ho Chi Minh City, Viet Nam

HOPE Research Center, Ho Chi Minh City, Viet Nam

Email: phuong.ltb@myduchospital.vn

History

- Received: Dec 21, 2020

- Accepted: Apr 27, 2021

- Published: May 30, 2021

DOI : 10.15419/bmrat.v8i5.671

\section{Check for updates}

\section{Copyright}

(- Biomedpress. This is an openaccess article distributed under the terms of the Creative Commons Attribution 4.0 International license.

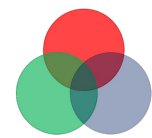

BioMedPress

The Open Access Publisher

\begin{abstract}
Background: Pre-implantation genetic testing for aneuploidy (PGT-A) has been using for years in embryoselection. However, this is an invasive method and may cause harm to the embryos. Therefore, time-lapse monitoring has been thought to be an alternative approach for embryo selection due to its efficiency. Up to now, several studies were investigating the relationship between the morphokinetic parameters and the embryo ploidy. However, the results are not consistent. This study aims to evaluate the correlation between morphokinetic parameters and PGT-A results. Methods: This retrospective cohort study was conducted at IVFMD Phu Nhuan, My Duc Phu Nhuan Hospital, between September 2018 and June 2019. Patients undergoing PGT-A due to advanced maternal age, repeated implantation failure or recurrent miscarriage and having embryo cultured under time-lapse monitoring were included. Patients with the re-thawing embryo for PGT-A were not eligible. The time from insemination to the pronuclear appearing (tPN), the onset of two to eight-cell divisions (t2 to t8) and the duration of the second cell cycle (cc2, t3-t2) were observed. Results: There were 39 patients included in the study, with mean age of $36.4 \pm 5.7$ years. A total of 110 blastocysts were biopsied. Amongst them, 63 embryos (57.3\%) were euploidy (group 1), and 47 embryos (42.7\%) were aneuploidy (group 2). There was no significant difference between euploid, and aneuploid embryos regarding all morphokinetic parameters, including tPN, t2, t3, t4, t5, cc2, and $\mathrm{t} 8(7.2 \pm 1.5$ hours vs. $7.4 \pm 1.6$ hours; $25.0 \pm 2.8$ hours vs. $25.6 \pm 3.2$ hours; $35.8 \pm$ 3.6 hours vs. $36.9 \pm 3.3$ hours; $37.5 \pm 4.4$ hours vs. $38.3 \pm 4.3$ hours; $49.2 \pm 5.52$ hours vs. 49.9 \pm 6.2 hours; $10.7 \pm 2.6$ hours vs. $11.2 \pm 1.7$ hours; and $55.7 \pm 6.4$ hours vs. $58.1 \pm 7.4$ hours, respectively). Conclusion: In this study, we found no difference in the morphokinetic parameters between euploid and aneuploid embryos.
\end{abstract}

Key words: aneuploidy, morphokinetic parameters, PGT-A, time-lapse system

\section{INTRODUCTION}

The selection of an embryo with the highest potential to implant is the top priority of many IVF centers. Up to now, the conventional method for embryo selection is morphology assessment. The cleavage-stage embryo's morphology was evaluated by the number of blastomeres, blastomere size, and the fragmentation degree. Other characteristics, such as multinucleation or the abnormal zona pellucida, also contribute to this evaluation ${ }^{1}$. At the blastocyst stage, the blastocoel expansion, the number, shape, and cohesion of cells within the inner cell mass, and the trophectoderm are used for the assessment. Some studies showed that these morphology parameters predict the clinical outcomes ${ }^{2,3}$. However, the correlation between embryo morphology and its implantation potential is relatively weak ${ }^{2,4}$.

Aneuploidy is a critical genetic factor that could influence human reproductive ability. Although morphological evaluation has been the primary method to choose an embryo, it was shown that aneuploid embryos are able to reach high morphological scores ${ }^{5}$. Therefore, pre-implantation genetic testing for aneuploidy (PGT-A) has been used for embryo selection $^{6,7}$. PGT-A has been indicated for patients at high-risk aneuploidy, such as advanced maternal age, repeated implantation failure, and recurrent miscarriage ${ }^{8,9}$. The biopsied materials were collected from the polar bodies ${ }^{10}$, the cleavage-blastomeres ${ }^{11}$, and the trophectoderm (TE) cells ${ }^{12}$. Among these biopsied materials, the blastocyst biopsy is the most common. The blastocyst biopsy provides more cells for genetic analysis while decreasing the invasiveness of the biopsy technique. However, the blastocyst biopsy requires extended culture, and investment in a laser system.

On the other hand, the biopsy of polar bodies only contains maternal DNA, and does not represent the embryo ploidy. In contrast, the biopsy of cleavageblastomere is an invasive technique, which affects the development, and the embryo implantation poten- 
tial ${ }^{10,13}$. There were controversies about the efficiency of PGT-A in IVF cycles ${ }^{9,14,15}$. The recent randomized controlled trials (RCT) study showed that PGT-A does not improve overall pregnancy outcomes in all women. There was a significant increase in the ongoing pregnancy rate among women with advanced maternal age ${ }^{16}$. Other concerns about the PGT-A are that this technique requires experienced embryologists, and modern equipment, incurs high costs, and precludes patients from performing a fresh embryo transfer cycle. Besides, the prolonged culture to blastocyst stage may be a less viable choice for older women or patients who had reduced ovarian reserve ${ }^{17}$. Due to these reasons, there is an increasing interest in the non-invasive method for embryo assessment.

The introduction of time-lapse monitoring (TLM) has allowed the observation of ongoing events during the pre-implantation stage ${ }^{18}$. This system is a non-invasive method that provides more information about the behavior of embryos. The morphokinetic parameters are helpful to predict blastocyst formation, implantation potential, and clinical outcomes $^{3,5}$. Besides, TLM could be used as another tool for the women in whom PGT-A could not be performed. Up to now, several studies have investigated the relationship between the morphokinetic parameters and the embryo ploidy. However, the results are not consistent among studies. The publications of Minasi et al. (2016), Mumusoglu et al. (2017), and Kimelman et al. (2019) reported a significant association with morphokinetic parameters and embryo ploidy status ${ }^{5,19,20}$. Nevertheless, Campbell et al. (2013) and Rienzi et al. (2015) found that no difference between aneuploid and euploid embryos with kinetic behaviors ${ }^{21,22}$.

The data of the association between embryo morphokinetic parameters and embryo ploidy status has been limited on Asian women with infertility. Therefore, the objective of this study was to determine whether aneuploid embryos display the morphokinetic parameters that were significantly different from euploid embryos. If any parameters were established, a further objective was to evaluate the correlation between the morphokinetic parameters and the PGT-A results of Vietnamese patients with a high risk of aneuploidy.

\section{METHODS}

\section{Study design}

This was a retrospective study conducted at IVFMD Phu Nhuan, My Duc Phu Nhuan Hospital, Ho Chi
Minh City, Viet Nam, between September 2018 and June 2019. The institutional ethics committee approved this study of My Duc Hospital (14/19/ĐĐBVMĐ, dated 30 September 2019).

\section{Study population}

Patients having indications for ART treatment and underwent PGT-A due to advanced maternal age, repeated implantation failure or recurrent miscarriage, and having embryo culture under TLM were included. Patients with the re-thawing embryo for PGTA were not eligible for the study.

\section{IVF protocol}

All participants underwent controlled ovarian hyperstimulation with a gonadotropin-releasing hormone $(\mathrm{GnRH})$ antagonist protocol. Recombinant folliclestimulating hormone dose (Merck, Germany) was 150-300 IU/day, depending on age, anti-Müllerian hormone levels, body mass index, and ovarian response. Follicle development was monitored using ultrasound, estradiol and progesterone levels. When the mean diameter of at least two follicles was $\geq 17 \mathrm{~mm}$, hCG (Merck, Germany) or GnRH agonist (Merck, Germany) was administered ${ }^{23}$. Thirty-six hours after the trigger, the oocyte cumulus complexes were retrieved by trans-vaginal ultrasound-guided aspiration. Upon retrieval, oocyte cumulus complexes were collected and cultured at $37^{\circ} \mathrm{C}, 6 \% \mathrm{CO}_{2}$, and $5 \% \mathrm{O}_{2}$ in the incubator (Origio, Denmark). After that, oocyte denudation was performed by enzymatic digestion with hyaluronidase (SAGE, Denmark) and mechanical pipetting. Fertilization was conducted by intracytoplasmic sperm injection (ICSI) at $39-41$ hours after the trigger. All embryos were individually cultured until the blastocyst stage in the time-lapse incubator (ASTEC, Japan) at $37^{\circ} \mathrm{C}, 6 \% \mathrm{CO}_{2}$, and $5 \% \mathrm{O}_{2}$. A fertilization check was performed at 16 - 18 hours after insemination. The morphology of cleavage-stage and blastocyst was evaluated at $64-68$ hours and 112 - 116 hours, respectively, after ICSI. Embryo classification was based on the Istanbul consensus (Alpha Scientists in Reproductive Medicine and ESHRE Special Interest Group Embryology, 2011).

\section{Blastocyst biopsy}

Embryo biopsied was performed on day 5. The blastocyst was breached of the zona pellucida (ZP) using a laser. This procedure allowed to creation of an opening in the ZP. After that, biopsy samples contained 5 - 6 TE cells were aspirated by a biopsy pipette (Origio, Denmark) under a laser-assisted cut (RI Saturn 5 
- UK). The TE cells were then washed in phosphatebuffered saline (PBS) with 1\% Polyvinylpyrrolidone (PVP) solution (Merck, Germany), stored in the microcentrifuge tubes (Merck, Germany) containing 2 $\mu$ L PBS (Merck, Germany), and were then genetically analyzed through the Karyolite Bobs technique. After the biopsy, the embryo was individually frozen by the vitrification method (Cryotech, Japan).

The Karyolite Bobs technique was carried out according to the manufacturer's protocol. The biopsied materials were amplified with biotin-labeled dNTP mix for 60 - 90 mins. After that, the unbound biotinlabeled dNTPs were removed, and the samples were hybridized to the bead at $52{ }^{\circ} \mathrm{C}$ in a shaking incubator for 16 hours. The signal was detected by the Luminex instrument. The data processing and aneuploidy detection were performed by dedicated software ${ }^{23}$. The PGT-A result showed the ploidy of all biopsied blastocyst was sent to the IVF laboratory. According to the result, an euploidy embryo was selected to transfer.

\section{Morphokinetic evaluation}

The embryo was assessed based on the morphology and morphokinetic parameters. All events of the preimplantation stage embryo were observed via Astec time-lapse incubator. The images of embryos cultured in the Astec time-lapse incubator were captured every 15 minutes (Figure 1). All the morphokinetic parameters of the biopsied embryo were assessed before receiving the PGT-A results. The recorded time points were selected based on the described parameters of Basile et al. (2014) ${ }^{24}$, included: The time from insemination to the pronuclear appearing ( $t P N$ ), the onset of two to eight-cell divisions ( $t 2$ to $t 8$ ), and the duration of the second cell cycle (cc2, $\mathrm{t} 3$ - $\mathrm{t} 2$ ). Besides, the abnormal cleavage characteristic was also recorded at the morphokinetic evaluation time and included: direct cleavage (DC- a single blastomere divided directly from 1 to 3 cells less than 5 hours); reverse cleavage (RVLV- an embryo decrease of the number during division) and MNB (multinucleated blastomeres) ${ }^{5}$.

\section{Statistical analysis}

Patients' characteristics and clinical outcomes were analyzed using descriptive statistics. Continuous variables were summarized as mean \pm standard deviation (SD), and categorical variables were presented as frequency (proportion). Biopsied embryos were divided into two groups: euploidy or aneuploidy, based on PGT-A. Morphokinetic parameters were compared between 2 groups by Student's test. All statistical analyses were performed using the R statistical package ( $\mathrm{R}$ version 3.5.0, R Foundation for Statistical Computing, Vienna, Austria). P-value $<0.05$ was considered to indicate statistical significance.

\section{RESULTS}

Between September 2018 and June 2019, a total of 39 women with 110 biopsied blastocysts were included in this study. The baseline characteristics of patients were shown in Table 1. The mean female age and BMI were $36.4 \pm 5.7$ years old and $22.0 \pm 2.7 \mathrm{~kg} / \mathrm{m}^{2}$. Among them, 24 (61.5\%) patients had advanced maternal age, $11(28.2 \%)$ patients had a recurrent miscarriage, and $4(10.3 \%)$ patients had repeated implantation failure.

The detail of ovarian stimulation was summarized in Table 2. The mean number of retrieved oocytes was $13.6 \pm 7.9$, while the mean number of good quality blastocyst was $3.2 \pm 2.4$ and the mean biopsied blastocyst was $3.1 \pm 1.9$ (Table 3 ). A total of 110 blastocysts was biopsied on day 5/6. Of the 110 analyzed embryos, 63 were euploid, and 47 were aneuploid. Among aneuploid embryos, 17 (36.2\%) had monosomy, 14 (29.8\%) had trisomy, and 16 (34\%) experienced complex abnormalities (more than one abnormal chromosome).

There was no significant difference between euploid and aneuploid embryos according to the morphokinetic parameters, such as $\mathrm{tPN}(7.2 \pm 1.5$ hours vs. 7.4 \pm 1.6 hours, $\mathrm{p}>0.05)$; $\mathrm{t} 2(25.0 \pm 2.8$ hours vs. $25.6 \pm$ 3.2 hours, $\mathrm{p}>0.05) ; \mathrm{t} 3(35.8 \pm 3.6$ hours vs. $36.9 \pm$ 3.3 hours, $\mathrm{p}>0.05)$; 4 ( $37.5 \pm 4.4$ hours and $38.3 \pm$ 4.3 hours, $\mathrm{p}>0.05) ; \mathrm{t} 5(49.2 \pm 5.5$ hours $v s .49 .9 \pm$ 6.2 hours, $\mathrm{p}>0.05) ; \mathrm{cc} 2(10.7 \pm 2.6$ hours $v s .11 .2 \pm$ 1.7 hours, $\mathrm{p}>0.05)$, and $\mathrm{t} 8(55.7 \pm 6.4$ hours $v s .58 .1$ \pm 7.4 hours, $\mathrm{p}>0.05)$ (Table 4 ).

\section{DISCUSSION}

This current study has combined the PGT-A technique and time-lapse monitoring to assess the correlation between the embryo ploidy status and morphokinetic parameters on infertility Vietnamese women. The study results demonstrated no significant difference between euploid and aneuploid blastocysts regarding the morphokinetic parameters $(\mathrm{tPN}, \mathrm{t} 2, \mathrm{t} 3, \mathrm{t} 4$, $t 5, t 8, c c 2$ ).

Some studies have shown that chromosomal abnormalities are the most common causes of poor clinical outcomes in IVF 25,26 . Over the past decade, ploidy analysis for the embryo has become popular. Some studies found that PGT-A improves the clinical outcome, such as increasing pregnancy rates, reducing abortion rates, higher birth rates, and lower malformation rates $8,9,14$. However, others suggested that 


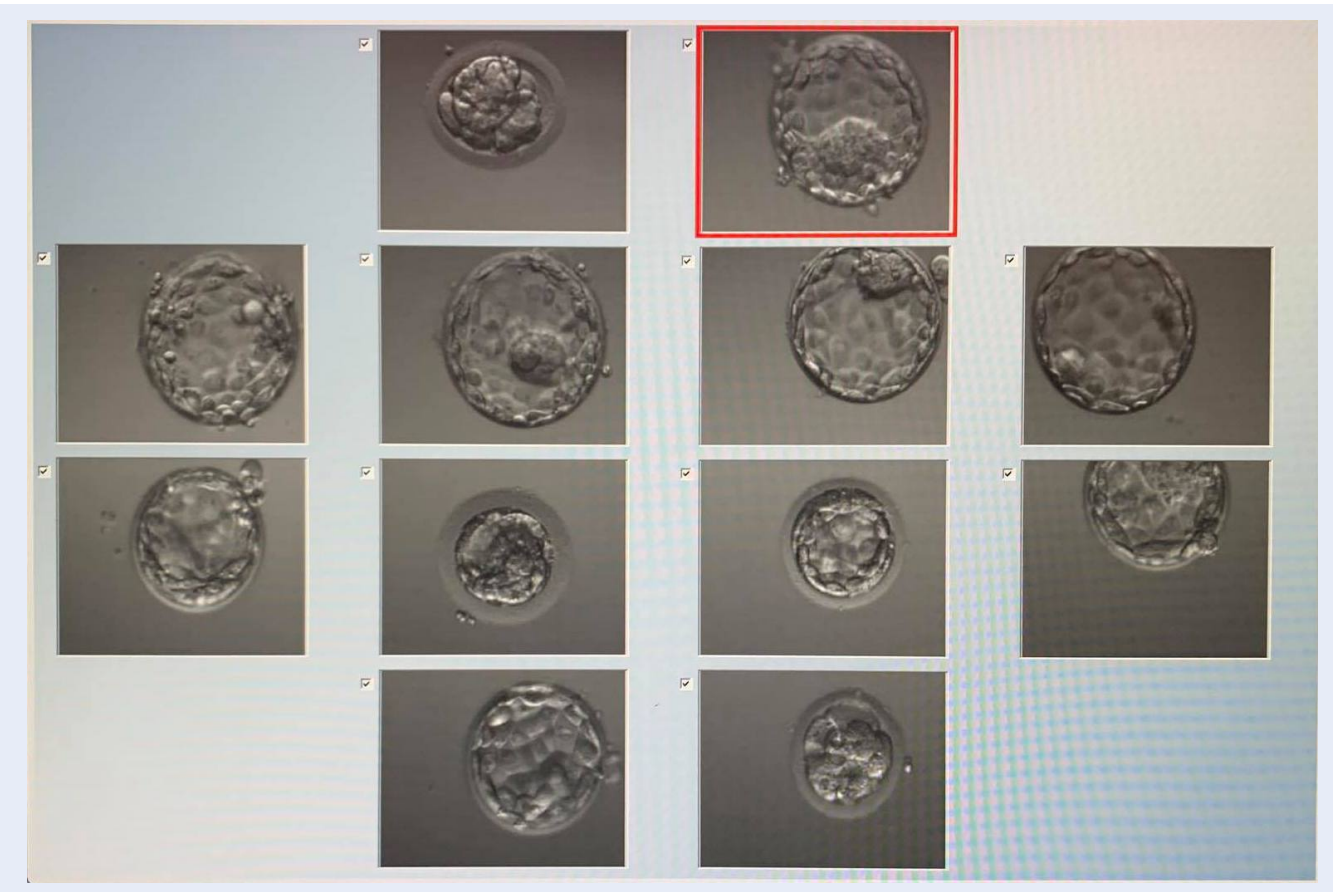

Figure 1: Embryos were monitored by Astec time-lapse system (IVFMD Phu Nhuan). The embryo morphology and morphokinetic parameters were continuously assessed from the oocytes to the blastocyst stage.

Table 1: The baseline characteristics

\begin{tabular}{lc}
\hline The baseline characteristics & $\mathrm{N}=39$ \\
\hline Age (years) & $36.4 \pm 5.7$ \\
BMI $\left(\mathrm{kg} / \mathrm{m}^{2}\right)$ & $22.0 \pm 2.7$ \\
AMH $(\mathrm{ng} / \mathrm{mL})$ & $3.2 \pm 2.3$ \\
AFC $(\mathrm{n})$ & $13.0 \pm 8.3$ \\
Duration of infertility (years) & $3.6 \pm 4.6$ \\
\hline Infertility type, $\mathbf{n}(\%)$ & $06(15.4)$ \\
\hline Primary & $33(84.6)$ \\
\hline Secondary & \\
\hline Number of IVF attempts, $\mathbf{n}(\%)$ & $25(64.1)$ \\
\hline 1 & $07(17.9)$ \\
\hline 2 & $07(17.9)$ \\
\hline
\end{tabular}

Data were shown as mean $\pm \mathrm{SD}$ or number $(\%)$ 
Table 2: The ovarian stimulation results

\begin{tabular}{lc}
\hline Parameters & $\mathbf{N}=\mathbf{3 9}$ \\
\hline Baseline FSH (IU) & $257.4 \pm 61.4$ \\
Duration of stimulation (days) & $10.1 \pm 3.5$ \\
Total FSH dose(IU) & $2679.5 \pm 1192.3$ \\
E2 trigger (pmol/L) & $3250.9 \pm 2652.6$ \\
P4 trigger (pmol/L) & $1.0 \pm 1.2$ \\
Endometrial thickness on trigger day $(\mathrm{mm})$ & $11.0 \pm 1.8$ \\
\hline
\end{tabular}

\section{Table 3: Embryonic outcomes}

\begin{tabular}{lc}
\hline Parameters & $\mathrm{N}=39$ \\
Number of oocytes retrieved (n) & $13.6 \pm 7.9$ \\
Number of MII oocytes (n) & $10.9 \pm 6.7$ \\
Number of 2PN (n) & $9.2 \pm 5.8$ \\
2PN rate (\%) & $86.2 \pm 15.1$ \\
Number of blastocyst (n) & $5.3 \pm 3.6$ \\
Blastocyst formation rate (\%) & $61.0 \pm 24.0$ \\
Number of good quality blastocyst - G1 + G2 (n) & $3.2 \pm 2.4$ \\
Good quality blastocyst rate (\%) & $62.9 \pm 33.4$ \\
Number of biopsied blastocyst (n) & $3.1 \pm 1.9$ \\
\hline
\end{tabular}

Table 4: The morphokinetic parameters of ploidy and aneuploidy

\begin{tabular}{ccccc}
\hline Parameters & $\begin{array}{r}\text { Aneuploidy } \\
(\mathbf{N}=\mathbf{4 7})\end{array}$ & $\begin{array}{c}\text { Ploidy } \\
(\mathbf{N}=\mathbf{6 3})\end{array}$ & $\begin{array}{c}\text { Between-group difference } \\
(\mathbf{9 5 \%} \mathbf{C I})\end{array}$ & $\mathbf{p}$ \\
tPN & $7.4 \pm 1.6$ & $7.2 \pm 1.5$ & $0.20(-0.42,0.81)$ & 0.52 \\
T2 & $25.6 \pm 3.2$ & $25.0 \pm 2.8$ & $0.55(-0.62,1.73)$ & 0.35 \\
T3 & $36.9 \pm 3.3$ & $35.8 \pm 3.6$ & $1.15(-0.17,2.47)$ & 0.09 \\
T4 & $38.3 \pm 4.3$ & $37.5 \pm 4.4$ & $0.77(-0.90,2.45)$ & 0.36 \\
T5 & $49.9 \pm 6.2$ & $49.2 \pm 5.5$ & $0.73(-1.55,3.01)$ & 0.53 \\
T8 & $58.1 \pm 7.4$ & $55.7 \pm 6.4$ & $2.41(-0.28,5.12)$ & 0.08 \\
CC2 & $11.2 \pm 1.7$ & $10.7 \pm 2.6$ & $0.47(-0.34,1.28)$ & 0.25 \\
\hline
\end{tabular}

Data were shown as mean \pm SD; p-values and $95 \%$ CI were estimated by Student's T-Test.

PGT-A did not benefit good-prognosis patients ${ }^{16}$ and women with decreased ovarian reserve. This method has been revealed that blastocyst biopsy affects the developmental competence of embryos. Besides, the implantation potential is negatively affected by the biopsied TE cell number in blastocysts with a low TE morphological score ${ }^{27}$. The recent development of genetic technologies has also increased the sensitivity of detecting mosaicism. Indeed, many mosaic embryos are discarded despite their potential for the gradual development of healthy babies ${ }^{28}$. In some situations, the biopsy for patients owning poor prognosis with fewer cleavage-stage embryos or insufficient quality blastocysts could not be performed. In this circumstance, the optimization of non-invasive technologies is essential to select an early embryo stage with the highest developmental potential. Thus, there is an increasing interest in assessing the correlation 
between the morphokinetic parameters and embryo ploidy status.

Our study shows similar results with Zhang J et al. (2017) that morphokinetic parameters such as tPNf, $\mathrm{t} 2, \mathrm{t} 3, \mathrm{t} 4, \mathrm{t} 5, \mathrm{t} 8, \mathrm{t} 9, \mathrm{tcom}, \mathrm{tM}, \mathrm{tSB}, \mathrm{tB}, \mathrm{tEB}, \mathrm{CC} 1, \mathrm{CC} 2$, $\mathrm{CC} 3, \mathrm{~S} 2, \mathrm{~S} 3, \mathrm{t} 5-\mathrm{t} 2$, and $\mathrm{tB}-\mathrm{tSB}$ showed no significant difference in euploid embryos compared to aneuploidy $^{27}$. Campbell A et al. (2013) indicated that no significant difference in the timing of early events in development ( $\mathrm{t} 2, \mathrm{t} 3, \mathrm{cc} 2$, and $\mathrm{s} 2$ ) between euploid and aneuploid embryo; however, they found the association between the $\mathrm{tSB}$ and $\mathrm{tB}$ parameters and the aneuploid risk ${ }^{21}$. Similarly, Rienzi L et al. could not find a correlation between 16 morphokinetic characteristics and aneuploidy rates ${ }^{22}$.

On the other hand, Chawla $\mathrm{M}$ et al. (2015) pointed out that $\mathrm{tPNf}, \mathrm{t} 2, \mathrm{t} 5, \mathrm{CC} 2, \mathrm{CC} 3$, and $\mathrm{t} 5$ - $\mathrm{t} 2$ differed significantly between aneuploidy and euploidy ${ }^{11}$. Similar to this study, the retrospective cohort study of Basile $\mathrm{N}$ et al. (2014) also found that chromosomally normal and abnormal embryos have different kinetic behavior. The data analysis identified cc3 (t5t2) parameter as the most relevant variables related to normal chromosomal content ${ }^{24}$. The publication of Carmen $\mathrm{N}$ et al. (2016) showed that $\mathrm{t} 3$ and $\mathrm{t} 5$-t 2 parameters were efficient ones associated with complex aneuploidy embryos ${ }^{29}$. In other research, Mumusoglu S et al. (2016) reported that only five time-lapse parameters as $\mathrm{t} 9, \mathrm{tM}, \mathrm{tSB}, \mathrm{tB}$, and $\mathrm{tEB}$ were initially noted to be significantly different among euploid and aneuploid blastocysts ${ }^{20}$. Moreover, Minasi M et al. analyzed the morphokinetic parameters of 928 blastocysts. They found that euploid embryos required shorter intervals to start (tSB), complete $(\mathrm{tB})$, expand (tEB), and hatch (tHB) blastocysts compared to aneuploid embryos 5 .

To our knowledge, this is the first study that was carried out on Vietnamese women with a high risk of aneuploidy. The strength of our study was the data come from a center experienced in blastocyst biopsy. Some limitations could be considered. Firstly, the study has a small sample size. Secondly, irregular division such as direct cleavage, chaotic division, reverse cleavage, or uneven cleavage was not assessed. Finally, the morphokinetic parameters at the morula and blastocyst stages were not analyzed.

\section{CONCLUSION}

Our study found no significant difference in the morphokinetic parameters between euploid and aneuploid embryos when assessing tPN, t2, t3, t4, t5, t8, and cc2 parameters. A study with larger sample size is necessary to demonstrate the correlation between the morphokinetic parameters and PGT-A results on infertility Asian women. The correlation between the time-lapse parameters and embryo ploidy status is still conflicted. The time-lapse parameters cannot be used as a substitute for PGT-A to select euploid embryos accurately. However, the time-lapse parameters provide valuable information for improving embryo selection and productive outcomes. Therefore, the combination of time-lapse parameters and PGT-A could enhance the selection of euploid embryos with the highest implantation potential.

\section{ABBREVIATIONS}

AMH: Anti-Muller hormone

ART: Assisted reproductive technology

BMI: Body mass index

FSH: Follicle stimulating hormone

ICSI: Intracytoplasmic sperm injection

IVF: In-vitro fertilization

PGT-A: Pre-implantation Genetic Testing - Aneuploidy

TE: Trophectoderm cell

TLM: time-lapse monitoring

ZP: zona pellucida

\section{ACKNOWLEDGMENTS}

This study was performed at IVFMD Phu Nhuan, My Duc Phu Nhuan Hospital. We are thankful to our colleagues who positively assisted to complete this study.

\section{AUTHOR'S CONTRIBUTIONS}

PTBL wrote the manuscript. AHL revised the manuscript. VQD planned and designed the experiments. LMTN collected and analyzed the data. All authors read and approved the final manuscript.

\section{FUNDING}

None

\section{AVAILABILITY OF DATA AND MATERIALS}

Data and materials used and/or analyzed during the current study are available from the corresponding author on reasonable request.

\section{ETHICS APPROVAL AND CONSENT TO PARTICIPATE}

This study was conducted in accordance with the amended Declaration of Helsinki. This study was approved by the institutional ethics committee of My Duc Hospital (14/19/ĐĐ-BVMĐ, dated 30 September 2019), and all participants provided written informed consent. 


\section{CONSENT FOR PUBLICATION}

Not applicable.

\section{COMPETING INTERESTS}

The authors declare that they have no competing interests.

\section{REFERENCES}

1. Medicine ASIR, Embryology ESIG. Istanbul consensus workshop on embryo assessment: proceedings of an expert meeting. Reprod Biomed Online. 2011;22(6):632-46. PMID: 21481639. Available from: 10.1016/j.rbmo.2011.02.001.

2. den Abbeel EV, Balaban B, Ziebe S, Lundin K, Cuesta MJ, Klein BM. Association between blastocyst morphology and outcome of single-blastocyst transfer. Reprod Biomed Online. 2013;27(4):353-61. PMID: 23953585. Available from: 10.1016/ j.rbmo.2013.07.006.

3. Ahlström A, Westin C, Wikland M, Hardarson T. Prediction of live birth in frozen-thawed single blastocyst transfer cycles by pre-freeze and post-thaw morphology. Hum Reprod. 2013;28(5):1199-209. PMID: 23477908. Available from: 10. 1093/humrep/det054.

4. Majumdar G, Majumdar A, Verma IC, Upadhyaya KC. Relationship Between Morphology, Euploidy and Implantation Potential of Cleavage and Blastocyst Stage Embryos. J Hum Reprod Sci. 2017;10(1):49-57. PMID: 28479756.

5. Minasi MG, Colasante A, Riccio T, Ruberti A, Casciani V, Scarselli F. Correlation between aneuploidy, standard morphology evaluation and morphokinetic development in 1730 biopsied blastocysts: a consecutive case series study. Hum Reprod. 2016;31(10):2245-54. PMID: 27591227. Available from: 10.1093/humrep/dew183.

6. Forman EJ, Hong KH, Ferry KM, Tao X, Taylor D, Levy B. In vitro fertilization with single euploid blastocyst transfer: a randomized controlled trial. Fertil Steril. 2013;100(1). PMID: 23548942. Available from: 10.1016/j.fertnstert.2013.02.056.

7. Scott RT, Upham KM, Forman EJ, Zhao T, Treff NR. Cleavagestage biopsy significantly impairs human embryonic implantation potential while blastocyst biopsy does not: a randomized and paired clinical trial. Fertil Steril. 2013;100(3):624-30. PMID: 23773313. Available from: 10.1016/j.fertnstert.2013.04. 039.

8. Rubio C, Bellver J, Rodrigo L, Castillón G, Guillén A, Vidal C. In vitro fertilization with preimplantation genetic diagnosis for aneuploidies in advanced maternal age: a randomized, controlled study. Fertil Steril. 2017;107(5):1122-9. PMID: 28433371. Available from: 10.1016/j.fertnstert.2017.03.011.

9. Verpoest W, Staessen C, Bossuyt PM, Goossens V, Altarescu G, Bonduelle M. Preimplantation genetic testing for aneuploidy by microarray analysis of polar bodies in advanced maternal age: a randomized clinical trial. Hum Reprod 2018;33(9):1767-76. PMID: 30085138. Available from: 10. 1093/humrep/dey262.

10. Cimadomo D, Capalbo A, Ubaldi FM, Scarica C, Palagiano A, Canipari R. The Impact of Biopsy on Human Embryo Developmental Potential during Preimplantation Genetic Diagnosis. BioMed Res Int. 2016;2016:7193075. PMID: 26942198. Available from: 10.1155/2016/7193075.

11. Chawla M, Fakih M, Shunnar A, Bayram A, Hellani A, Perumal V. Morphokinetic analysis of cleavage stage embryos and its relationship to aneuploidy in a retrospective time-lapse imaging study. J Assist Reprod Genet. 2015;32(1):69-75. PMID: 25395178. Available from: 10.1007/s10815-014-0372-3.

12. Griffin DK, Ogur C. Chromosomal analysis in IVF: just how useful is it? Reproduction. 2018;156(1):29-50. PMID: 29945889. Available from: 10.1530/REP-17-0683.

13. Scott RT, Upham KM, Forman EJ, Hong KH, Scott KL, Taylor D. Blastocyst biopsy with comprehensive chromosome screening and fresh embryo transfer significantly increases in vitro fertilization implantation and delivery rates: a randomized controlled trial. Fertil Steril. 2013;100(3):697-703. PMID: 23731996. Available from: 10.1016/j.fertnstert.2013.04.035.

14. Phuong L, Thuc V, Quan P, Anh L, Vinh D, Huyen N. Selecting euploid embryo for transfer by preimplantation genetic testing for aneuploidy improved clinical outcomes in patients with advanced maternal age. Biomed Res Ther. 2019;6(12):3541-9. Available from: 10.15419/bmrat.v6i12.581.

15. Rubio C, Simón C, Vidal F, Rodrigo L, Pehlivan T, Remohí J. Chromosomal abnormalities and embryo development in recurrent miscarriage couples. Hum Reprod. 2003;18(1):182-8. PMID: 12525464. Available from: 10.1093/humrep/deg015.

16. Munné S, Kaplan B, Frattarelli JL, Child T, Nakhuda G, Shamma $\mathrm{FN}$, et al. Preimplantation genetic testing for aneuploidy versus morphology as selection criteria for single frozen-thawed embryo transfer in good-prognosis patients: a multicenter randomized clinical trial. Fertil Steril. 2019;112(6). PMID: 31551155. Available from: 10.1016/j.fertnstert.2019.07.1346.

17. Marca AL, Minasi MG, Sighinolfi G, Greco P, Argento $C$, Grisendi V. Female age, serum antimüllerian hormone level, and number of oocytes affect the rate and number of euploid blastocysts in in vitro fertilization/intracytoplasmic sperm injection cycles. Fertil Steril. 2017;108(5). PMID: 28987789. Available from: 10.1016/j.fertnstert.2017.08.029.

18. Huang TT, Chinn K, Kosasa T, Ahn HJ, Kessel B. Morphokinetics of human blastocyst expansion in vitro. Reprod Biomed Online. 2016;33(6):659-67. PMID: 27665055. Available from: 10.1016/j.rbmo.2016.08.020.

19. Kimelman D, Confino R, Okeigwe I, Lambe-Steinmiller J, Confino E, Shulman LP. Assessing the impact of delayed blastulation using time lapse morphokinetics and preimplantation genetic testing in an IVF patient population. J Assist Reprod Genet. 2019;36(8):1561-9. PMID: 31385120. Available from: 10.1007/s10815-019-01501-1.

20. Mumusoglu S, Yarali I, Bozdag G, Ozdemir P, Polat M, Sokmensuer LK. Time-lapse morphokinetic assessment has low to moderate ability to predict euploidy when patient- and ovarian stimulation-related factors are taken into account with the use of clustered data analysis. Fertil Steril. 2017;107(2). PMID: 27939508. Available from: 10.1016/j.fertnstert.2016.11.005.

21. Campbell A, Fishel S, Bowman N, Duffy S, Sedler M, Thornton $S$. Retrospective analysis of outcomes after IVF using an aneuploidy risk model derived from time-lapse imaging without PGS. Reprod Biomed Online. 2013;27(2):140-6. PMID: 23683847. Available from: 10.1016/j.rbmo.2013.04.013.

22. Rienzi L, Capalbo A, Stoppa M, Romano S, Maggiulli R, Albricci L. No evidence of association between blastocyst aneuploidy and morphokinetic assessment in a selected population of poor-prognosis patients: a longitudinal cohort study. Reprod Biomed Online. 2015;30(1):57-66. PMID: 25458852. Available from: 10.1016/j.rbmo.2014.09.012.

23. Vuong L, Pham L, Huynh B, Nguyen QN, Ho TM, Norman RJ. Live Birth Rate After Transfer of Fresh or Frozen Poor Quality Day-3 Embryos Only. Fertil Reprod. 2019;01(4):161-8. Available from: 10.1142/S266131821950018X.

24. Basile N, Nogales MC, Bronet F, Florensa M, Riqueiros M, Rodrigo L. Increasing the probability of selecting chromosomally normal embryos by time-lapse morphokinetics analysis. Fertil Steril. 2014;101(3):699-704. PMID: 24424365. Available from: 10.1016/j.fertnstert.2013.12.005.

25. Campbell A, Fishel S, Bowman N, Duffy S, Sedler M, Hickman CF. Modelling a risk classification of aneuploidy in human embryos using non-invasive morphokinetics. Reprod Biomed Online. 2013;26(5):477-85. PMID: 23518033. Available from: 10.1016/j.rbmo.2013.02.006.

26. Vanneste $\mathrm{E}$, Voet $\mathrm{T}$, Caignec $\mathrm{CL}$, Ampe M, Konings $\mathrm{P}$, Melotte C. Chromosome instability is common in human cleavagestage embryos. Nat Med. 2009;15(5):577-83. PMID: 19396175. Available from: $10.1038 / \mathrm{nm} .1924$

27. Zhang J, Tao W, Liu H, Yu G, Li M, Ma S. Morphokinetic parameters from a time-lapse monitoring system cannot accu- 
rately predict the ploidy of embryos. J Assist Reprod Genet. 2017;34(9):1173-8. PMID: 28676910. Available from: 10.1007/ s10815-017-0965-8.

28. Zaninovic N, Irani M, Meseguer M. Assessment of embryo morphology and developmental dynamics by time-lapse microscopy: is there a relation to implantation and ploidy? Fer- til Steril. 2017;108(5):722-9. PMID: 29101997. Available from: 10.1016/j.fertnstert.2017.10.002.

29. Nogales MDC, Bronet F, Basile N, Martínez EM, Liñán A, Rodrigo L. Type of chromosome abnormality affects embryo morphology dynamics. Fertil Steril. 2017;107(1). PMID: 27816230. Available from: 10.1016/j.fertnstert.2016.09.019. 